Pecvnia, Monográfico (2009), pp. 55-76

\title{
Las existencias
}

\section{Cristina Gutiérrez López}

cristina.gutierrez.lopez@unileon.es Universidad de León

Fac. de Ciencias Económicas y Empresariales

Campus de Vegazana, $\mathrm{s} / \mathrm{n}$ 24071 León (España)

\section{CONCEPTO Y NORMATIVA}

La normativa internacional reguladora del tratamiento contable de las existencias se recoge en las siguientes normas: NIC "2. Existencias", NIC "11. Contratos de construcción", NIC 32 y "39. Commodities", NIC "40. Inmuebles de inversión distintos a los adquiridos para su venta en el curso normal de la explotación", NIC "41. Activos biológicos relacionados con la actividad agrícola".

En el caso español, la Norma de Registro y Valoración (NRV) $\mathrm{n}^{\circ}$ "10. Existencias del Nuevo Plan General Contable" (en adelante, NPGC) es la destinada a estos activos corrientes (NRV 12 en el Plan de Pymes), si bien debería analizarse en combinación con la NRV "14. Ingresos por ventas y prestación de servicios". 
Las existencias son activos corrientes:

- Poseídos para ser vendidos en el curso normal de la explotación.

- En proceso de producción de cara a esa venta.

- En forma de materiales o suministros, para ser consumidos en el proceso de producción o suministro de servicios ${ }^{1}$.

- También bienes comprados y almacenados para revender (mercaderías); terrenos $u$ otras inversiones inmobiliarias que se tienen para ser vendidas a terceros; productos terminados o en curso y materiales para usarse en el proceso productivo.

Por tanto, habitualmente se trata de elementos que se encuentran físicamente en la empresa, salvo los recibidos en consigna, los recibidos en depósito, aquellos en que la compra está pendiente de aprobación o si la empresa no asume los riesgos y beneficios; situaciones todas ellas en que la ubicación física de las existencias no se corresponde con el control económico. También puede tratarse de unidades que estén en poder del proveedor, o unidades en tránsito hacia la empresa, siempre que los riesgos se hayan transferido a ésta. De igual modo, se incluyen las unidades en consigna, depósito, etc. en manos de los clientes, y también aquellas en tránsito hacia éstos, si los riesgos aún no se han transferido.

En definitiva, el momento de su alta o baja en el balance será aquel en que se produzca el cambio de propiedad. No obstante, el NPGC da prioridad al control económico, es decir, al cambio de responsabilidad para responder por la integridad de esos elementos. Se trata pues de responder a la siguiente cuestión: ¿quién asumiría las pérdidas en caso de pérdida, deterioro o similar?

Del tratamiento de las existencias en el NPGC no se infieren grandes cambios respecto al Plan 1990, si exceptuamos la consideración de los servicios dentro del concepto de existencias y la no aceptación del LIFO como fórmula de coste, tal que las modificaciones no son tan relevantes como en otros elementos. Las operaciones con envases $\mathrm{y} / \mathrm{o}$ embalajes con facultad de devolución no se han modificado.

De acuerdo al Plan 1990, era posible valorar ciertas materias primas y consumibles por una cantidad y valor fijos, circunstancia no admitida con el NPGC. 
En cuanto a la elaboración del Balance de situación, el NPGC indica que deberán desglosarse en el balance las existencias de ciclo corto y largo. Además, los anticipos entregados a proveedores se incluirán como parte del activo corriente en la agrupación de Existencias (y no dentro de Acreedores comerciales). Sin embargo, los anticipos recibidos de clientes se incluirán con los Acreedores comerciales en el pasivo corriente.

\section{VALORACIÓN INICIAL}

Las existencias se reconocerán en el balance desde que la entidad tenga los riesgos y ventajas inherentes a la propiedad de las mismas.

Su valoración inicial se realizará al coste, formado por los costes derivados de su adquisición y transformación, así como aquellos otros en que se incurra hasta que se encuentren en su condición y ubicación actuales.

- Si las existencias se compran, el criterio de referencia será el precio de adquisición, formado por:

Importe facturado por el vendedor

- Descuentos

+ Gastos adicionales hasta que los bienes estén ubicados para su venta, si son responsabilidad del comprador (frente a la consideración del Plan 1990, que los incluía hasta que los bienes estuvieran en el almacén)

+ Intereses incorporados al nominal de los débitos ${ }^{2}$

De esta definición destacan tres aspectos novedosos:

1. Todos los descuentos o rebajas que se conozcan en el momento de la adquisición reducirán el precio de adquisición. Por tanto, el registro de los descuentos en cuentas diferentes sólo se producirá

2 Por el contrario, en la normativa internacional, siguiendo la NIC "23. Costes por intereses", éstos no aumentarán el valor de las existencias, sino que serán gastos. La coincidencia entre las NIC y NPGC sí se produce en el caso de las existencias de ciclo largo. 
cuando se concedan en fechas posteriores, ya sea por volumen (609. Rappel sobre compras), incumplimiento de las condiciones, retrasos, devoluciones, etc. (608. Devoluciones de compras y operaciones similares) o pronto pago (606. Descuento sobre compras por pronto pago). En este último caso, en lugar de reflejar un ingreso financiero se opta ahora por reconocer un menor gasto de explotación ${ }^{3}$. Así, el destino de las tres cuentas será formar parte del resultado de explotación de la Cuenta de pérdidas y ganancias.

2. Los intereses que surjan en operaciones a crédito no forman parte del precio de adquisición, reflejándose como gastos financieros (662. Intereses de deudas). La excepción serán aquellos intereses asociados a débitos con vencimiento a un plazo no superior a un año, y sin tipo de interés contractual. En estos casos, no reducirán el precio de adquisición, y la deuda se reflejará a valor nominal (por tanto, es de aplicación la NRV 9 sobre pasivos financieros, en el caso de débitos por operaciones comerciales).

3. Heredada de las normas sobre inmovilizado (NRV 2.1), es obligatorio incluir los gastos financieros asociados a las "existencias de ciclo largo". Así, formarán parte de la valoración los gastos financieros en las existencias que necesiten un período de tiempo superior a un año para estar en condiciones de ser vendidas (aplicable también al cálculo del coste de producción) ${ }^{4}$.

Por tanto, se excluyen del cálculo del precio de adquisición los costes de almacenamiento posterior (se entiende, a la disposición de las unidades para la venta), gastos generales de administración que no

En la transición al NPGC, si la empresa decidiera aplicar el nuevo criterio para el tratamiento de los descuentos por pronto pago de forma retroactiva, debería registrar un ajuste en el valor de las existencias (que aumentaría) contra una cuenta de reservas. No obstante, se trata de un supuesto opcional. De optar por él, deberá incorporarse una nota en la memoria sobre "Aspectos derivados de la transición a las nuevas normas contables", facilitando una explicación sobre las principales diferencias entre los criterios contables aplicados en el ejercicio anterior y los actuales, y la cuantificación del impacto que produce esta variación de criterios contables en el patrimonio neto de la empresa (conciliación entre patrimonio neto a 31-12-07 y patrimonio neto con los criterios del NPGC a 1-1-08).

4 En el primer ejercicio que se inicie a partir de 2008 procederá cargar la cuenta de existencias (grupo 3) por el importe de los gastos financieros no activados en su momento, contra el abono de (11) Reservas por ajustes. No obstante, puede obviarse esta anotación si se opta por aplicar el régimen transitorio, que recoge que la empresa puede optar por no aplicar con efectos retroactivos el criterio de capitalización de gastos financieros incluido en la NRV 10.1. 
contribuyen a dar a las existencias su condición y ubicación actuales, y los costes de comercialización. Tampoco se incluyen las diferencias de cambio que hayan surgido directamente de su adquisición reciente en moneda extranjera (pero sí las que surgen por financiación).

\section{Ejemplo $\mathbf{n}^{0}$ 1.- Compra de mercaderías con pago aplazado (I)}

1-1-X1 una empresa compra mercaderías por 1.000€, aplazando el pago a 6 meses. El IVA de la operación es el 16\%. Se considera que el tipo de interés de créditos de riesgo similar es del $4 \%$.

Se pide: Registro contable.

\section{Solución.-}

De acuerdo a la NRV 10, el precio de adquisición estará formado:

Importe facturado por el vendedor

- Descuentos

- Intereses incorporados al nominal de los débitos

+ Gastos adicionales hasta la ubicación para su venta

Y de forma simultánea, en operaciones a crédito, la deuda con los proveedores se registrará como un pasivo financiero (NRV 9), inicialmente al valor razonable (precio de la transacción o valor razonable de la contraprestación entregada más costes de la transacción), registrándose posteriormente a coste amortizado.

$$
\frac{1.160}{(1+i)^{0,5}}=1.137,47
$$

$$
\begin{aligned}
& \text { 977,47 } 600 \text { Compras mercaderías } \\
& 1.137,47-160 \\
& 160,00472 \text { H.P. IVA soportado } \\
& 22,53662 \text { Intereses de deudas }
\end{aligned}
$$

400 Proveedores

$$
1.160 /(1+\mathrm{i})^{0,5}=1.137,47
$$

400 Proveedores

$$
1.137,47\left[(1,04)^{0,5}-1\right]=
$$$$
1.137,47\left[(1+0,04)^{0,5}\right]-1.137,47
$$

Sin embargo, en el cálculo del precio de adquisición, podrán incluirse los intereses incorporados al nominal de los débitos cuando el vencimiento no sea a más de un año, no haya tipo de interés contractual y el efecto de no actualizar los flujos de efectivo no sea significativo; esto supone que tales intereses no reduzcan el precio de adquisición y, por tanto, no se reflejen por separado como gastos financieros.

Simétricamente, la deuda con los proveedores puede valorarse a Valor nominal si es una operación comercial, el plazo no es superior a un año, no hay tipo de interés contractual y el efecto de no actualizar los flujos no es significativo. 


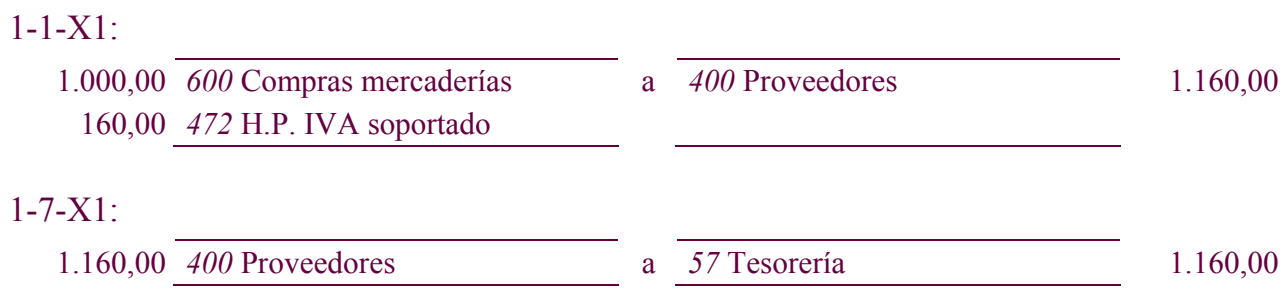

\section{Ejemplo $n^{0}$ 2.- Compra de mercaderías con pago aplazado (II)}

1-1-X1 una empresa compra mercaderías cuyo precio al contado es de 1.000€; debido al aplazamiento en el pago (6 meses), deberá pagar $200 €$ adicionales. El IVA de la operación es el $16 \%$.

Se pide: Registro contable.

\section{Solución.-}

Dado que hay tipo de interés contractual, el criterio de valoración ha de ser valor razonable.

\begin{tabular}{|c|c|c|c|c|}
\hline \multirow{4}{*}{$\begin{array}{r}1.000,00 \\
160,00 \\
200,00\end{array}$} & \multicolumn{3}{|c|}{$1.160(1+i)^{0,5}=1.360 \rightarrow i=\mathrm{TIE}=37,45 \%$} & \multirow{3}{*}{$1.160,00$} \\
\hline & 600 Compras mercaderías & $\mathrm{a}$ & 400 Proveedores & \\
\hline & 472 H.P. IVA soportado & & & \\
\hline & 662 Intereses de deudas & $\mathrm{a}$ & $\begin{array}{l}400 \text { Proveedores } \\
1.160\left[(1+0,3745)^{0,5}-1\right]\end{array}$ & 200,00 \\
\hline $1.360,00$ & $\begin{array}{c}400 \text { Proveedores } \\
1.160+200\end{array}$ & $\mathrm{a}$ & 57 Tesorería & $1.360,00$ \\
\hline
\end{tabular}

\section{Ejemplo n ${ }^{0}$ 3.- Compra de mercaderías con pago aplazado (III)}

1-6-X1 una empresa compra mercaderías cuyo precio al contado es de 2.000€, concediendo el proveedor un descuento promocional de $400 €$ incluido en la factura, y cancelándose un anticipo entregado a éste previamente por 500€. No se pacta ningún tipo de interés contractual, y se acepta la factura a 15 meses. El tipo de interés de mercado para operaciones similares es del 4\%. El IVA de la operación es el 16\%.

Se pide: Registro contable.

\section{Solución.-}

Pese a que no hay tipo de interés contractual, la operación tiene un vencimiento superior a un año, por lo que debe registrarse inicialmente a valor razonable y posteriormente a coste amortizado. 
Valoración:

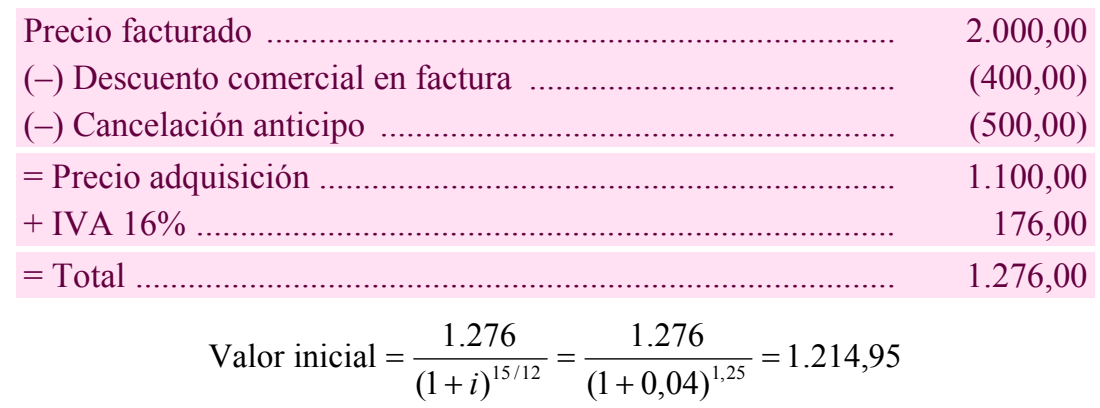

01-06-X1:

\begin{tabular}{|c|c|c|}
\hline & & \\
\hline 176,00 & & \\
\hline
\end{tabular}

Para el cálculo del coste amortizado será preciso reflejar los intereses $(61,05 €)$, que elevarán el valor del pasivo financiero (proveedores), periodificando tales intereses según su devengo en X1 y X2.

Registro de intereses a 31-12-X1 $=\left[1.214,95(1+i)^{7 / 12}\right]-1.214,95=28,12$ 31-12-X1:

$$
\begin{aligned}
& 28,1 2 \longdiv { 6 6 2 \text { Intereses de deudas } } \text { a } \\
& \text { Proveedores }(\text { coste amortizado })=1.214,95+28,12=1.243,07
\end{aligned}
$$

1-9-X2:

$$
32,93 \begin{gathered}
\text { Intereses }=\left[1.243,07(1+i)^{8 / 12}\right]-1.243,07=32,93 \\
\text { a62 Intereses de deudas }
\end{gathered}
$$

Pago:

$$
\text { Coste amortizado }=1.243,07+32,93=1.276
$$

$1.276,00 \underline{400 \text { Proveedores }}$ a

\section{Ejemplo n ${ }^{0}$ 4.- Existencias de ciclo largo}

Una empresa se dedica al secado de jamones, teniendo el proceso una duración de 24 meses. 1-1-X1 ha comprado 400 jamones a un matadero a 60€/ud. Para financiar esta compra ha solicitado un crédito a 2 años con un tipo de interés del 5\% anual, pagadero cada 31 de diciembre.

Se pide: Reflejo contable.

\section{Solución.-}

1-1-X1 Compra de materias primas: 


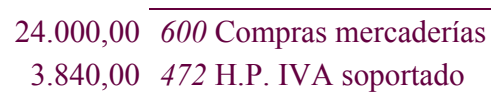

Financiación obtenida:

31-12-X1 Intereses primer año y reclasificación del préstamo:

\begin{tabular}{|c|c|c|c|}
\hline $1.200,00$ & $\begin{array}{l}662 \text { Intereses de deudas } \\
5 \% 24.000\end{array}$ & $\mathrm{a}$ & 572 Banco c/c \\
\hline 0,0 & $\begin{array}{l}170 \text { Deudas a largo plazo con } \\
\text { entidades de crédito }\end{array}$ & $\mathrm{a}$ & $\begin{array}{l}520 \text { Deudas a corto plazo con } \\
\text { entidades de crédito }\end{array}$ \\
\hline
\end{tabular}

Valor de los jamones a 31-12-X1:

Consumo de materias primas

$=$ Total

31-12-X2 Intereses segundo año:

$1.200,00662$ Intereses de deudas $\overline{572 \mathrm{Banco} \mathrm{c} / \mathrm{c}} \quad 1.200,00$ $5 \% 24.000$

Productos terminados (considerando que no se ha vendido ninguna unidad):

$26.400,00350$ Productos terminados $25.200+1.200$ a 712 Variación de existencias de productos terminados

- En las existencias sometidas a transformación, se calculará el coste de producción, formado por los costes directamente relacionados con las unidades producidas (materiales y mano de obra directa) y una asignación sistemática de gastos generales (costes indirectos), fijos y variables. Los costes variables se asignan a cada unidad de producción en función del uso real de los medios de producción. Los fijos se reparten según la capacidad normal de trabajo de los medios de producción. Los costes totales no se incrementan por un bajo de nivel de producción; en períodos de producción anormalmente alta, los costes indirectos fijos asignados a cada unidad producida disminuyen de modo que las existencias no se valoren por encima del coste real. Los costes indirectos que no se imputan a las existencias se reconocen como gasto del ejercicio. 
La novedad en la definición del coste de producción respecto al Plan 1990 es que se ha incluido la consideración de incorporar al coste de producción los costes indirectos fijos teniendo en cuenta el nivel de utilización de la capacidad normal de trabajo de los medios de producción ${ }^{5}$ (método de imputación racional); así, el coste por subactividad no puede ser incorporado al coste de producción de las existencias, y se considerará como gasto del ejercicio.

Sin embargo, extrapolando las especificaciones recogidas por la NIC 2, no se asignan al coste de las existencias sino que se reconocen como gasto, las pérdidas por deterioro, las cantidades anormales de desperdicio de materiales, mano de obra u otros costes de producción, los costes generales de administración que no estén relacionados con la producción de existencias (por ejemplo, los costes de funcionamiento de un departamento financiero), ni, como se comentó previamente, los costes de subactividad.

En cuanto a los costes por intereses, según la NIC 23 su tratamiento preferente es considerarlos gasto del ejercicio, siendo el tratamiento permitido la capitalización ${ }^{6}$.

\section{Ejemplo $\mathrm{n}^{0}$ 5.- Cálculo del coste de producción}

Una empresa dedicada a la fabricación de muebles de oficina calcula el coste de producción de 50 mesas de oficina a partir de los siguientes datos, sabiendo que la capacidad normal estimada de producción son 60 mesas:

5 Tomando como referencia la NIC 2, la capacidad normal es la producción que se espera conseguir en circunstancias normales, considerando el promedio de varios ejercicios o temporadas, y teniendo en cuenta la pérdida de capacidad que resulta de las operaciones previstas de mantenimiento. La cantidad de coste indirecto fijo distribuido a cada unidad de producción no se incrementará como consecuencia de un nivel bajo de producción, ni por la capacidad ociosa. Los costes indirectos no distribuidos se reconocerán como gastos del ejercicio en que han sido incurridos. Los costes indirectos variables se distribuirán a cada unidad de producción sobre la base del nivel real de uso de los medios de producción.

6 Los costes por intereses directamente atribuibles a la adquisición o producción de activos cualificados (los que requieren un periodo de tiempo sustancial para estar listos para el uso o la venta) deben ser capitalizados. Estos costes son los que podrían haberse evitado si no se hubiera efectuado ningún desembolso sobre ese activo. Aquellas existencias que se fabrican rutinariamente o que se producen en grandes cantidades de forma repetitiva y necesitan períodos cortos de tiempo para su producción, no se consideran activos cualificados, ni tampoco las mercaderías. 


\begin{tabular}{|c|c|}
\hline Consumo de materias primas y otros $c$ & $18.100,00$ \\
\hline Mano de obra directa ................... & $21.000,00$ \\
\hline Costes fijos indirectos de fabricación & \\
\hline Gastos diversos ................ & $1.200,00$ \\
\hline 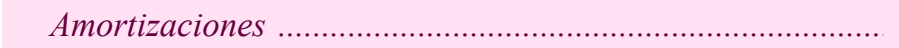 & $2.400,00$ \\
\hline Costes variables indirectos de fabricación & \\
\hline Mano de obra indirecta & - \\
\hline Materiales indirectos & \\
\hline
\end{tabular}

Se pide: Cálculo del coste de producción.

Solución.-

Coste de producción:

\begin{tabular}{|c|c|}
\hline mo de materia & $18.100,00$ \\
\hline 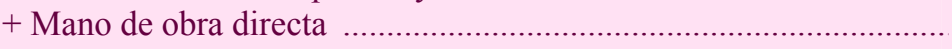 & $21.000,00$ \\
\hline + Costes variables indirectos de fabricación & $3.980,00$ \\
\hline + Costes fijos indirectos de fabricación $(3.600 € / 60$ udes $) \times 50$ udes & $3.000,00$ \\
\hline Total & $46.080,00$ \\
\hline
\end{tabular}

El coste de subactividad es de $600 €$ (3.600-3.000), calculado por diferencia entre los costes fijos indirectos totales y los que se han imputado según la capacidad normal de producción de la empresa. No forma parte del coste de producción y se lleva a resultados.

- El NPGC hace una referencia expresa a la prestación de servicios. Así, para el cálculo del coste de las existencias de un prestador de servicios servirán los criterios relativos al precio de adquisición, coste de producción y métodos de asignación de valor. Las existencias incluirán el coste de producción de los servicios en tanto aún no se hayan reconocido los ingresos por prestación de servicios correspondientes, conforme a la NRV 14 y permitiendo una mejor correlación de ingresos y gastos en el cálculo del resultado. No se incluye la mano de obra ni otros costes relacionados con la venta, ni tampoco los costes del personal de administración general (son gasto del ejercicio).

Se hace así alusión a aquellas empresas cuya actividad principal consiste en prestar servicios que necesitan un largo período de tiempo, antes de estar en condiciones de prestarse efectivamente al cliente, tal que es normal que al cierre del ejercicio el servicio no se haya terminado, habiéndose reconocido los gastos en que la empresa ha incurrido, sin haber registrado aún ingreso alguno. Para evitar ese desfase entre ingresos y gastos, es posible activar como existencias los costes de producción de los servicios hasta que no se reconozca el ingreso por la prestación efectiva. 


\section{Ejemplo n ${ }^{0}$ 6.- Prestación de servicios}

Una empresa está diseñando la página Web para un cliente, no habiendo acabado al cierre del ejercicio y sin reconocer ingreso alguno. Los costes asumidos hasta el momento son:

\begin{tabular}{|c|c|}
\hline Mano de obra (diseñador, programador informático) & $2.500,00$ \\
\hline Material de oficina consumido & 300,00 \\
\hline Dietas (desplazamiento para reuniones con cliente) & 420,00 \\
\hline Costes indirectos distribuibles ............. & 310,00 \\
\hline Costes indirectos no distribuibles & 890,00 \\
\hline Costes de comercialización ........ & $1.050,00$ \\
\hline
\end{tabular}

Se pide: Valorar las existencias en forma de servicios.

\section{Solución.-}

Los costes indirectos no distribuibles, así como los de comercialización (y los generales, si los hubiera) no forman parte del coste del servicio:

\begin{tabular}{|c|c|}
\hline Mano de obra & $2.500,00$ \\
\hline Material de oficina & 300,00 \\
\hline Dietas ...................... & 420,00 \\
\hline Costes indirectos distribuibles & 310,00 \\
\hline Coste del servicio en curso & $3.530,00$ \\
\hline
\end{tabular}

$\begin{array}{llll}3.530,00 & \text { a } & \begin{array}{l}710 \text { Variación de existencias de } \\ \text { productos en curso }\end{array} & 3.530,00\end{array}$

No se pueden incluir en el valor de las existencias finales los costes indirectos no distribuibles ni los costes de comercialización $=890+1.050=1.940 €$ :

$\begin{array}{llll}1.940,00 & \text { a Gastos } & 1.940,00\end{array}$

- Fórmulas de coste o métodos de asignación de valor.

El NPGC indica que deberá realizarse una valoración individualizada, siempre que sea posible, como ocurrirá en el caso de bienes producidos y segregados para proyectos específicos. En caso contrario, con carácter general se aplicará un precio medio ponderado ${ }^{7}$, salvo que sea más conveniente para la gestión de la empresa el método FIFO. Por

7 El NPGC no explicita si ese precio medio ponderado debe calcularse de forma global para el período considerado o deberá obtenerse después de cada entrada de existencias en los almacenes. Es previsible que posteriores normas que desarrollen el Plan señalen aspectos más concretos a este respecto. 
tanto, se establece una jerarquía de criterios y queda descartada la opción de utilizar el método LIFO. No se dice nada respecto al coste estándar y el método de minoristas, sí incluidos en la NIC 2.

La prohibición de aplicar el método LIFO hace necesario realizar un ajuste en la contabilidad de aquellas empresas que vinieran utilizándolo, en el primer ejercicio iniciado a partir de 1-1-08, normalmente aumentando el valor de existencias, tal que: aplicación NPGC

Adicionalmente, tal y como se recoge en el marco conceptual, una vez adoptado un criterio ha de mantenerse en el tiempo y aplicarse a existencias de naturaleza y uso similares para la empresa (principio de uniformidad), aunque podrá modificarse si cambian los supuestos que motivaron su elección.

En la NIC 2 se indica también que si las existencias no son de naturaleza similar o tienen distinto uso para la entidad, puede justificarse el uso de fórmulas distintas. La ubicación geográfica ( $\mathrm{y}$, por tanto, el distinto tratamiento fiscal) no es motivo suficiente.

- Además de las consideraciones anteriores, deben tenerse en cuenta dos situaciones adicionales:

- Existencias que se han financiado en moneda extranjera (véase el capítulo relativo a diferencias de cambio).

- Subvenciones, donaciones o legados: a) Subvenciones, donaciones o legados que se reciben en forma de existencias, que se valorarán por el valor razonable en el momento de su reconocimiento. $\mathrm{Si}$ dichas existencias se deterioran, se considerará un deterioro irreversible en la parte en la que hayan sido financiadas gratuitamente. b) Subvención monetaria recibida que está destinada a la financiación de la adquisición de existencias, de modo que dichas existencias se darán de alta en el patrimonio de la empresa por el precio de adquisición.

En ambos casos, la subvención se imputará a resultados como ingresos del ejercicio en que se produzca su enajenación, corrección valorativa por deterioro o baja en balance (NRV 18). 
Ejemplo n ${ }^{0}$ 7.- Recepción de un legado consistente en mercaderías

Una empresa dedicada a la construcción de muebles de diseño ha recibido en mayo del ejercicio X1 un legado de $200.000 \mathrm{~m}^{2}$ de madera de ébano, con un valor razonable de 150.000€. Al cierre del ejercicio, la mitad de la madera se había incorporado a productos terminados que se vendieron durante $X 1$.

Se pide: Registro (considerar Plan Pymes).

\section{Solución.-}

200.000,00 31 Materias primas

$\underline{ }$

132 Otras subvenciones, donaciones y legados $200.000,00$

Imputación a resultados del ejercicio:

$\begin{array}{ccc}100.000,00 & \begin{array}{c}132 \text { Otras subvenciones, } \\ \text { donaciones y legados }\end{array}\end{array}$

a
47 Otras subvenciones, donaciones
y legados transferidos al resultado del ejercicio

$100.000,00$

\section{VALORACIÓN POSTERIOR}

En un momento posterior a su reconocimiento en el balance, las existencias deberán figurar al menor valor entre el coste o el valor neto realizable (VNR), dado que el criterio general es que los activos no se valorarán en libros por importes superiores a lo que se espera obtener a través de su venta o uso, a efectos de no sobrevalorar el patrimonio.

El VNR ${ }^{8}$ será el importe neto que se espera realizar a partir de la venta de las existencias en el curso normal de la explotación, es decir, es un valor específico de la entidad, a diferencia del valor razonable, ya que al VNR se le da la categoría de estimación subjetiva y personalizada y no necesariamente contrastable con un valor razonable de mercado ${ }^{9}$.

8 En el concepto de coste (precio de adquisición o coste de producción) no se incluyen los costes de administración, distribución y venta del producto, por tanto, para que el precio de venta de mercado y coste sean comparables, a ese precio de venta hay que descontarle los costes de distribución y venta, obteniendo así el VNR, que se compara con el coste para aplicar el menor de los dos.

9 Según las NIC, las estimaciones del VNR deben tener en cuenta: a) fluctuaciones de precios o costes directamente relacionados con sucesos o hechos que hayan tenido lugar después del cierre del ejercicio, b) el propósito para el que se mantienen las existencias (por ejemplo, en existencias para cumplir los contratos de venta o de prestación de servicios, se basa en el precio fijado en el contrato). 
analizadas:

El cálculo del VNR dependerá del tipo de existencias

- Mercaderías: Precio estimado de venta - Costes necesarios para la venta

- Productos en curso: Precio estimado de venta (del producto terminado) - Costes necesarios para la venta - Costes estimados para concluir la producción

- Materias primas: Precio de reposición

En caso de que el VNR sea inferior al coste, procederá reflejar la correspondiente corrección valorativa, teniendo en cuenta dos aspectos:

- Para materiales consumibles en el proceso de producción, no habrá corrección si los productos a los que se incorporen pueden venderse por encima de su coste.

- En caso de existencias objeto de un contrato de venta en firme, si el precio acordado supera al conjunto de valor inicial y costes pendientes para ejecutar el contrato, no habrá corrección.

El incremento de valor de las existencias se producirá cuando las circunstancias que previamente causaron la rebaja del valor hayan dejado de existir, o cuando exista una clara evidencia de un incremento del VNR.

\section{Ejemplo $n^{0}$ 8.- Correcciones de valor (mercaderías)}

Información sobre existencias de mercaderías:

\begin{tabular}{|c|c|c|c|c|}
\hline Mercaderías & Udes. & $\begin{array}{c}\text { Coste adquisición } \\
(\boldsymbol{\epsilon} / \mathbf{u d})\end{array}$ & $\begin{array}{c}\text { Precio venta } \\
(\boldsymbol{\epsilon} / \mathbf{u d})\end{array}$ & $\begin{array}{c}\text { Coste de venta } \\
(\boldsymbol{\epsilon} / \mathbf{u d})\end{array}$ \\
\hline$A$ & 200 & 2 & 2,5 & 0 \\
\hline$B$ & 400 & 1,5 & 1,4 & 0,3 \\
\hline$C$ & 350 & 2,7 & 3,5 & 0 \\
\hline$D$ & 100 & 3 & 2,8 & 0 \\
\hline
\end{tabular}

Sobre la mitad de las unidades $D$ existe un contrato de venta en firme a un precio de 3,5€, siendo los costes estimados de su comercialización 0,05€/ud.

Se pide: Determinar las posibles correcciones valorativas. 


\section{Solución.-}

Considerando que las 4 mercaderías corresponden con productos diferentes y no relacionados, los cálculos deben realizarse por separado:

\begin{tabular}{|c|c|c|c|c|}
\hline \multicolumn{2}{|c|}{ Mercaderías } & Coste & VNR & ¿Deterioro? \\
\hline \multicolumn{2}{|r|}{$\mathrm{A}$} & $200 \mathrm{ud} \times 2 € / \mathrm{ud}=400 €$ & $200 \mathrm{ud} \times 2,5 € / \mathrm{ud}=500 €$ & No \\
\hline \multicolumn{2}{|r|}{ B } & $400 \mathrm{ud} \times 1,5 € / \mathrm{ud}=600 €$ & $400 \mathrm{ud} \times(1,4-0,3) € / \mathrm{ud}=440 €$ & $160 €$ \\
\hline \multicolumn{2}{|r|}{ C } & $350 \mathrm{ud} \times 2,7 € / \mathrm{ud}=945 €$ & $350 \mathrm{ud} \times 3,5 € / \mathrm{ud}=1.225 €$ & No \\
\hline \multirow{2}{*}{$\mathrm{D}$} & Con contrato & $50 \mathrm{ud} \times 3 € / \mathrm{ud}=150 €$ & $50 \mathrm{ud} \times(3,5-0,05) € / \mathrm{ud}=172,5 €$ & No \\
\hline & Sin & $150 €$ & $50 \mathrm{ud} \times 2,8 € / \mathrm{ud}=140 €$ & $10 €$ \\
\hline
\end{tabular}

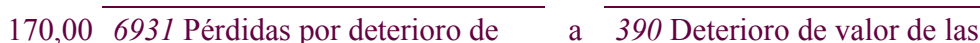
mercaderías $160+10$ mercaderías

Ejemplo n $^{0}$ 9.- Correcciones de valor (materias primas, productos en curso terminados)

Información sobre existencias de mercaderías:

\begin{tabular}{|c|c|c|c|c|}
\hline Partida & Udes. & $\begin{array}{c}\text { Coste adquisición } \\
(\boldsymbol{\epsilon})\end{array}$ & $\begin{array}{c}\text { Precio venta } \\
(\boldsymbol{\epsilon} / \mathbf{u d})\end{array}$ & $\begin{array}{c}\text { Coste de venta } \\
(\boldsymbol{\epsilon} / \mathbf{u d})\end{array}$ \\
\hline Mat. primas A & 500 & 250 & Precio de reposición $=0,4 € /$ ud & \\
\hline Prod. curso $A$ & 40 & 2.700 & & \\
\hline Prod. term. $A$ & 120 & 12.000 & 90 & 5 \\
\hline
\end{tabular}

La materia prima A se destina a la fabricación del producto terminado A.

Para terminar la fabricación del producto en curso A se estima serán precisos unos costes de $15 €$ por unidad de producto.

Se pide: Determinar las posibles correcciones valorativas.

\section{Solución.-}

Como el producto terminado A no puede venderse por encima del coste de fabricación, debe analizarse también la posible corrección valorativa para la materia prima y para el producto en curso.

* Producto terminado:

$$
\begin{aligned}
& \mathrm{VNR}=90-5=85 € / \mathrm{ud} \\
& \text { Coste }=12.000 € / 120 \mathrm{udes}=100 € / \mathrm{ud} . \\
& \text { Corrección valorativa }=120 \mathrm{udes} \times 15 € / \mathrm{ud}=1.800 €
\end{aligned}
$$

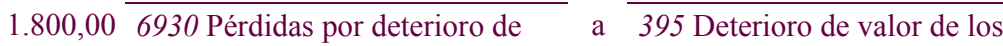
productos terminados y en curso productos terminados de fabricación

* Materias primas:

$\mathrm{VNR}=$ coste de reposición $=0,4 € / \mathrm{ud} \times 500$ udes $=200 €$

Corrección valorativa $=250-200=50 €$ 
50,00 6932 Pérdidas por deterioro de materias primas
391 Deterioro de valor de las materias primas
50,00

* Producto en curso A: si para terminar la producción de A en curso es estima incurrir en $15 € /$ ud, entonces:

$$
\begin{aligned}
& \mathrm{VNR}=40 \times(90-5-15)=2.800 € \\
& \text { Coste }=2.700 €
\end{aligned}
$$

Por tanto, no hay corrección valorativa.

\section{- Ventas y prestaciones de servicios}

El NPGC elimina toda referencia a las pérdidas irreversibles de las existencias, tal que en la regularización al cierre dicha pérdida, de haberse producido, se recogerá al reconocer el valor de las existencias finales reales.

Como se mencionó en las páginas iniciales, es preciso completar el tratamiento de las existencias con las normas referentes a los ingresos. Así, la NRV "14. Ingresos por ventas y prestaciones de servicios" se adapta de forma fiel a lo establecido en las normas internacionales ${ }^{10}$.

Por tanto, para la venta de existencias, son aplicables los criterios de la NRV 14, que indica que se reconocerán ingresos por el valor razonable de la contrapartida recibida, determinado a partir del precio acordado, que se reducirá por los descuentos y los intereses incorporados al nominal de los créditos (reflejados estos últimos pues como ingresos financieros). No obstante, dichos intereses podrán incluirse cuando se trate de operaciones a un plazo no superior a un año y en operaciones sin tipo de interés contractual, cuando el efecto de no actualizar los flujos de efectivo no sea significativo.

De este modo, en la valoración de las ventas los descuentos por pronto pago reciben un tratamiento distinto al Plan 1990. De igual modo, se introduce el criterio de valor razonable para el registro de los créditos por operaciones comerciales. En cuanto a los intereses por aplazamiento en el pago, la desaparición de los ingresos a distribuir en varios ejercicios hace que ahora se imputen a resultados como ingresos

10 NIC "18. Ingresos ordinarios", NIC "11. Contratos de construcción", NIC "20. Contabilización de las subvenciones del gobierno e información a revelar sobre ayudas gubernamentales", SIC "27. Evaluación del fondo económico de las transacciones que adoptan la forma legal de un arrendamiento", SIC "31. Ingresos ordinarios-Permutas de servicios de publicidad". 
financieros a medida que se devenguen y de acuerdo al método del TIE. La excepción a esa norma, como se comenta en el párrafo anterior, permitirá la valoración a valor nominal (opción no contemplada por las normas internacionales).

Además, la norma indica claramente los criterios para el reconocimiento de los ingresos por la venta de bienes, para lo que debe cumplirse un conjunto de requisitos:

- Transferencia de riesgos y beneficios al comprador, independientemente de su transmisión jurídica. No se producirá pues en ventas con pacto de recompra.

- Pérdida de la gestión corriente y el control de los bienes vendidos.

- Valoración fiable.

- Probabilidad de recibir los rendimientos económicos de la transacción (relacionada con la probabilidad de que se reciba la contraprestación o desaparezca cierta incertidumbre). Sobre este aspecto, el Cuadro 1 resume los ejemplos más habituales, recogidos por la NIC 18.

- Valoración fiable de los costes de la transacción. Así, siguiendo la necesaria correlación de ingresos y gastos que recoge el marco conceptual, los ingresos y gastos relacionados con una misma transacción o hecho deben reconocerse simultáneamente ${ }^{11}$.

Además de la venta de bienes, se hace referencia a la prestación de servicios, destacando que se reconocerán ingresos cuando el resultado de la transacción pueda estimarse con fiabilidad ${ }^{12}$, teniendo en cuenta el porcentaje de realización del servicio a la fecha de cierre del ejercicio.

11 A este respecto, la NIC 18 es más explícita, indicando que no se podrán reconocer ingresos si los gastos asociados a la venta no pueden medirse con fiabilidad, de modo que en el momento de registro de la venta, la contrapartida de la contraprestación recibida (efectivo o un derecho de cobro) será un pasivo, que posteriormente se trasladará a una cuenta de ingresos, cuando pueda realizarse con fiabilidad dicha valoración.

12 Según la NIC 18, pueden estimarse fiablemente dichos ingresos cuando se ha acordado con la otra parte del contrato cuáles son los derechos de cada parte, la contrapartida a intercambiar y la forma y los términos de pago. 
Cuando el resultado de la transacción que implique la prestación de servicios no pueda estimarse fiablemente, no puede reconocerse beneficio alguno, dado que se reconocerán ingresos sólo en la cuantía en que los gastos reconocidos se consideren recuperables. Es decir, el coste de producción del servicio que no esté terminado a fecha de cierre del ejercicio se activará como existencia, reconociéndose un ingreso (la variación de existencias) por la cifra de los costes incurridos, tal y como confirma la NRV 10.

\begin{tabular}{|l|l|}
\multicolumn{1}{|c|}{ Tipología de ventas } & \multicolumn{1}{|c|}{ Momento de reconocimiento del ingreso } \\
\hline Ventas facturadas, aún no entregadas & $\begin{array}{l}\text { El comprador adquiere la titularidad y reconoce } \\
\text { las condiciones de entrega, la entrega es } \\
\text { probable y los bienes están disponibles e } \\
\text { identificados }\end{array}$ \\
\hline Ventas con derecho limitado de devolución & $\begin{array}{l}\text { Bienes entregados, y el plazo de devolución ha } \\
\text { concluido }\end{array}$ \\
\hline Ventas en consigna & $\begin{array}{l}\text { El receptor de los bienes los ha vendido a un } \\
\text { tercero }\end{array}$ \\
\hline Ventas contra reembolso & Bienes entregados y cobro de la operación \\
\hline $\begin{array}{l}\text { Ventas con custodia (entrega cuando el } \\
\text { comprador realiza el pago final, tras una serie } \\
\text { de plazos) }\end{array}$ & \begin{tabular}{l} 
Bienes entregados y, por tanto, cobrados \\
\hline $\begin{array}{l}\text { Ventas a plazos } \\
\text { Momento de la venta (excepto intereses, que se } \\
\text { recogerán a medida que se devenguen) }\end{array}$ \\
\hline
\end{tabular}
\end{tabular}

El método del grado de avance o porcentaje de realización ${ }^{13}$ (en detrimento del contrato cumplido) calcula el grado de avance como la relación entre los costes incurridos al cierre del ejercicio y el coste total estimado del producto o servicio, siendo pues el volumen de ingresos obtenidos hasta el momento ese mismo porcentaje aplicado sobre el total presupuestado.

La empresa debe revisar las estimaciones del ingreso a recibir, a medida que el servicio se va prestando. La necesidad de esas revisiones indica que el desenlace o resultado de la operación no pueda ser estimado con fiabilidad.

13 Fiscalmente, este es el método más favorable, ya que permite diferir el pago de impuestos. No obstante, como contraviene el principio de devengo, para su aplicación se requiere la aprobación por parte de la administración. 


\section{Ejemplo $n^{0} 10$ - Ingresos por servicios}

Una empresa de consultoría recibe en el ejercicio X1 el encargo de elaborar un informe para otra entidad. El presupuesto de ingresos para el estudio es de 75.000€, comenzando en mayo de X1 y con una duración de 2 años. Se estima que los costes para el desarrollo del estudio serán 27.000€ en X1, 22.950 en X2 y 17.550 en X3.

Los ingresos imputables a cada ejercicio dependerán del porcentaje de costes incurridos.

Se pide:

a) Registro de la operación si se cumplen las condiciones para el reconocimiento de los ingresos

b) No se cumplen las condiciones para el reconocimiento de los ingresos pero los costes se consideran recuperables

c) No se cumplen las condiciones para el reconocimiento de los ingresos y los costes no se consideran recuperables

\section{Solución.-}

a) Se cumplen las condiciones para reconocer los ingresos

El resultado estimado será:

$$
\text { Ingresos }- \text { Costes }=75.000-(27.000+22.950+17.550)=7.500 €
$$

Para el ejercicio X1:

$$
\frac{\text { Costes incurridos }}{\text { Costes totales }}=\frac{27.000}{67.500}=40 \%
$$

Ingresos ejercicio X1:

$$
40 \% 75.000=30.000 €
$$

Resultado X1:

$$
30.000-27.000=3.000
$$

En la tabla siguiente se recogen los ingresos a contabilizar en cada período a través de

\begin{tabular}{|c|c|c|c|}
\hline $27.000,00$ & 6 Gastos & $\mathrm{a}$ & Tesorería/Pasivos \\
\hline $30.000,00$ & 43 Clientes, facturas pendientes & $\mathrm{a}$ & 705 Prestaciones de servicios \\
\hline
\end{tabular}
abonos de "705. Prestación de servicios":

\begin{tabular}{|c|c|c|c|c|c|}
\hline Ejercicio & $\begin{array}{c}\text { Costes } \\
\text { período }\end{array}$ & $\begin{array}{c}\text { Coste } \\
\text { acumulado }\end{array}$ & $\begin{array}{c}\text { \% } \\
\text { realizado }\end{array}$ & $\begin{array}{c}\text { Ingresos } \\
\text { del período }\end{array}$ & Resultado \\
\hline X1 & 27.000 & 27.000 & $40 \%$ & 30.000 & 3.000 \\
\hline X2 & 22.950 & 49.950 & $34 \%$ & 25.500 & 2.550 \\
\hline X3 & 17.550 & 67.500 & $26 \%$ & 19.500 & 1.950 \\
\hline Total & 67.500 & & $100 \%$ & 75.000 & 7.500 \\
\hline
\end{tabular}

Durante X1:

Durante X2: 


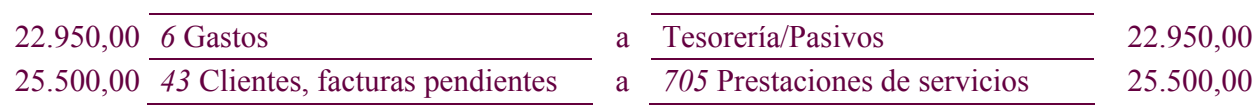

Durante X3:

\begin{tabular}{|c|c|c|c|}
\hline $17.550,00$ & 6 Gastos & $\mathrm{a}$ & Tesorería/Pasivos \\
\hline $19.500,00$ & 43 Clientes, facturas pendientes & $\mathrm{a}$ & 705 Prestaciones de servicios \\
\hline $75.000,00$ & Tesorería/Activos & $\mathrm{a}$ & 43 Clientes, facturas pendientes \\
\hline
\end{tabular}

b) No se cumplen las condiciones para el reconocimiento de los ingresos pero los costes se consideran recuperables

\begin{tabular}{|c|c|c|c|}
\hline Ejercicio & Costes período & Ingresos del período & Resultado \\
\hline X1 & 27.000 & 27.000 (Var.exist.) & 0 \\
\hline X2 & 22.950 & 22.950 (Var.exist.) & 0 \\
\hline X3 & 17.550 & $75.000-49.950=25.050$ (Var.exist.) & 7.500 \\
\hline
\end{tabular}

Durante X1:

$27.000,0 0 \longdiv { 6 \text { Gastos } }$ a

\section{1-12-X1:}

27.000,00 33 Productos en curso a 710 Var.exist. productos en curso

Durante X2:

$22.950,006$ Gastos

31-12-X2:

22.950,00 33 Productos en curso

Durante X3:

$17.550,0 0 \longdiv { 6 \text { Gastos } }$

$\begin{array}{ll}\text { Tesorería/Pasivos } & 17.550,00\end{array}$

Mayo X3: Final del trabajo:
$75.000,00$ Tesorería/Activos
705 Prestaciones de servicios
$75.000,00$
31-12-X3:
49.950,00 710 Var.exist. productos en curso $27.000+22.950$
33 Productos en curso
$49.950,00$

c) No se cumplen las condiciones para el reconocimiento de los ingresos y los costes no se consideran recuperables

En ese caso, los costes se registrarán en cada período correspondiente y los ingresos sólo cuando el servicio se haya completado (X3).

Durante X1:

$\begin{array}{lll}27.000,00 & \text { a Gastos } & \text { 27.000,00 }\end{array}$


Durante X2:

$22.950,00 \quad 6$ Gastos

a Tesorería/Pasivos

$22.950,00$

Durante X3:

$\begin{array}{ll}17.550,00 & \overline{6 \text { Gastos }} \\ 75.000,00 & \end{array}$

$\begin{array}{llr}\text { a } & \text { Tesorería/Pasivos } & 17.550,00 \\ \text { a } & 705 \text { Prestaciones de servicios } & 75.000,00\end{array}$

Además, la NRV 14 indica que no se reconocerá ningún ingreso por la permuta de bienes o servicios, por operaciones de tráfico, de similar naturaleza y valor. Es decir, cuando la contraprestación de una venta sea la recepción de bienes/servicios y no el efectivo, no podrá reconocerse ningún ingreso, si se trata de operaciones de tráfico y los bienes son de similar naturaleza y valor. Este tratamiento de las permutas coincide con lo establecido en la NIC 18 (en cuyo caso, sólo se hace referencia a la similitud en la naturaleza).

\section{Ejemplo $\mathrm{n}^{0}$ 11.- Permutas de existencias}

Una entidad dedicada a la compra-venta de material de oficina entrega a otra empresa del mismo sector un lote de archivadores valorados a 200€, recibiendo a cambio grapadoras cuyo valor de mercado supone $220 €$.

Se pide:

a) Registro del intercambio considerando que los elementos tienen naturaleza distinta.

b) Registro del intercambio considerando que los elementos tienen naturaleza similar.

Solución.-

a) Se reconoce un ingreso por el valor razonable de la mercancía recibida:
220,00 600 Compras de mercaderías
a 700 Ventas de mercaderías
220,00
$35,20472 \mathrm{HP}$, IVA soportado
a $477 \mathrm{HP}$, IVA repercutido
35,20

b) En este caso, cabe plantearse si es preciso realizar asiento contable, dado que no puede reconocerse ingreso alguno. Una alternativa (a la espera de futuras resoluciones del ICAC al respecto) sería:
200,00 302 Mercaderías, grapadoras
a 301 Mercaderías, archivadores
200,00

Por último, cuando existan dudas relativas al cobro de un importe previamente reconocido como ingresos por venta y/o prestación de servicios, la cantidad cuyo cobro se estime como improbable se registrará 
como un gasto por corrección de valor por deterioro y no como un menor ingreso.

\section{BIBLIOGRAFÍA}

GonzÁlez QUINTANA, M.J. y E. CAÑAdas Molina (2008) "Adaptación contable del tratamiento de las existencias a la normativa internacional", Partida Doble, núm. 199, pp. 32-46.

INTERNATIONAL ACCOUNTING STANDARDS BOARD (2006) Normas Internacionales de información financiera (NIIF), texto completo de las Normas Internacionales de Información Financiera emitidas a 1 de enero de 2006, traducción al español publicada por CISS-PRAXIS con la autorización de la IASCF.

MANZANO, F. y A.M. TORRE (2007) "El tratamiento contable de las existencias en las Normas Internacionales de Información Financiera", CISS Técnica Contable, núm. 701, pp. 63-71.

PRIETO, B. y J. MATA (2007) "Existencias, acreedores por operaciones comerciales y compras", CISS Técnica Contable, núm. 701, pp. 16-32.

Vacas Guerrero, C.; M.J. Bonilla Priego y C. AVILÉs Palacios (2007) "Tratamiento contable de las existencias en el BPGC", Partida Doble, núm. 193, pp. 26-37.

ZAMORA RAMíReZ, C. (Coord.) (2007) Análisis práctico y guía de implantación del nuevo PGC. Valencia: CISS. 